\title{
Sensor Arrays and Electronic Tongue Systems
}

\author{
Manel del Valle \\ Sensores y Biosensores del Grupo, Departamento de Química, Universitat Autonoma de Barcelona, Bellaterra, 08193 Barcelona, Spain
}

Correspondence should be addressed to Manel del Valle, manel.delvalle@uab.es

Received 1 September 2011; Accepted 11 October 2011

Academic Editor: Thiago R. L. C. Paixão

Copyright (C) 2012 Manel del Valle. This is an open access article distributed under the Creative Commons Attribution License, which permits unrestricted use, distribution, and reproduction in any medium, provided the original work is properly cited.

\begin{abstract}
This paper describes recent work performed with electronic tongue systems utilizing electrochemical sensors. The electronic tongues concept is a new trend in sensors that uses arrays of sensors together with chemometric tools to unravel the complex information generated. Initial contributions and also the most used variant employ conventional ion selective electrodes, in which it is named potentiometric electronic tongue. The second important variant is the one that employs voltammetry for its operation. As chemometric processing tool, the use of artificial neural networks as the preferred data processing variant will be described. The use of the sensor arrays inserted in flow injection or sequential injection systems will exemplify attempts made to automate the operation of electronic tongues. Significant use of biosensors, mainly enzyme-based, to form what is already named bioelectronic tongue will be also presented. Application examples will be illustrated with selected study cases from the Sensors and Biosensors Group at the Autonomous University of Barcelona.
\end{abstract}

\section{Introduction}

Recently, in our laboratories, we have been exploring a new concept in the work with chemical sensors and biosensors, which is the electronic tongue [1]. This new concept in sensor science entails the use of sensors with reduced selectivity, grouped in arrays with cross-response characteristics. Accompanying this, the processing of the complex-generated information is completed with appropriate chemometric tools, in order to fulfil an analytical application. Variants can be a classical quantitative determination, where a number of substances may be determined in a single, direct operation, or the determination of a substance in the presence of its interferents, provided their separation is not needed [2-4]. Also, the concept can provide more interesting applications, such as identification of varieties or establishing the membership to a group or class, when pattern recognition procedures are applied. The latter qualitative applications are of great interest, as they can be the basis of automated detection principles in fields like food, beverages, or pharmaceutics, where alternatives to the human expert are in demand [5]. It is also possible to correlate a characteristic or property, for example, a perception, to the contribution of the different species sensed. In our studies, the preferred chemometric tool for the processing of data has been artificial neural networks (ANNs) [6]. These are known to be powerful nonlinear modellers, applicable for quantitative and also qualitative applications. In this sense, our approach is doubly biomimetic; firstly, the use of groups of sensors with cross-response is the sensing scheme in the taste buds of animals, and, secondly, employing ANNs which are parallel information processing tools inspired in the animal nervous system, whose maximum expression is the human brain.

The agreed definition of an "electronic tongue," as defined by significant research groups working in this topic can be summarized as [7] "an analytical instrument comprising an array of non-specific, poorly selective chemical sensors with partial specificity (cross sensitivity) to different compounds in a solution, coupled to an appropriate chemometric tool for data processing," or the recent IUPAC report on the topic defines it as [8] "a multisensor system, which consists of a number of low-selective sensors and uses advanced mathematical procedures for signal processing based on Pattern Recognition and/or Multivariate data analysis-Artificial Neural Networks (ANNs), Principal Component Analysis (PCA), and so forth.”

With respect to the type of sensors that can be used in an electronic tongue, practically all the main families of chemical sensors have been used to form the sensor array, namely, potentiometric, voltammetric, resistive, gravimetric, 
TABLE 1: Examples of different families of sensors used to form the sensor array in an electronic tongue.

\begin{tabular}{|c|c|c|c|}
\hline Sensor family & Research group & Example & Reference \\
\hline $\begin{array}{l}\text { Electrochemical, } \\
\text { potentiometric }\end{array}$ & Legin (Russia) & $\begin{array}{l}\text { Determination of heavy metals with an } \\
\text { array of chalcogenide membrane sensors }\end{array}$ & {$[30]$} \\
\hline $\begin{array}{l}\text { Electrochemical, } \\
\text { ISFETs }\end{array}$ & Bratov (Spain) & $\begin{array}{l}\text { Determination of several ions for water } \\
\text { characterization }\end{array}$ & {$[31]$} \\
\hline $\begin{array}{l}\text { Electrochemical, } \\
\text { voltammetric }\end{array}$ & Winquist (Sweden) & $\begin{array}{l}\text { Characterization of waters with an array } \\
\text { of noble metals }\end{array}$ & {$[32]$} \\
\hline Electrical, resistive & Mattoso (Brazil) & Conducting polymer sensors & {$[33]$} \\
\hline Optical & Mc.Devitt (USA) & Microspheres with immobilized dyes & {$[34]$} \\
\hline Gravimetric & Gardner (UK) & Surface acoustic wave (SAW) sensors & {$[35]$} \\
\hline
\end{tabular}

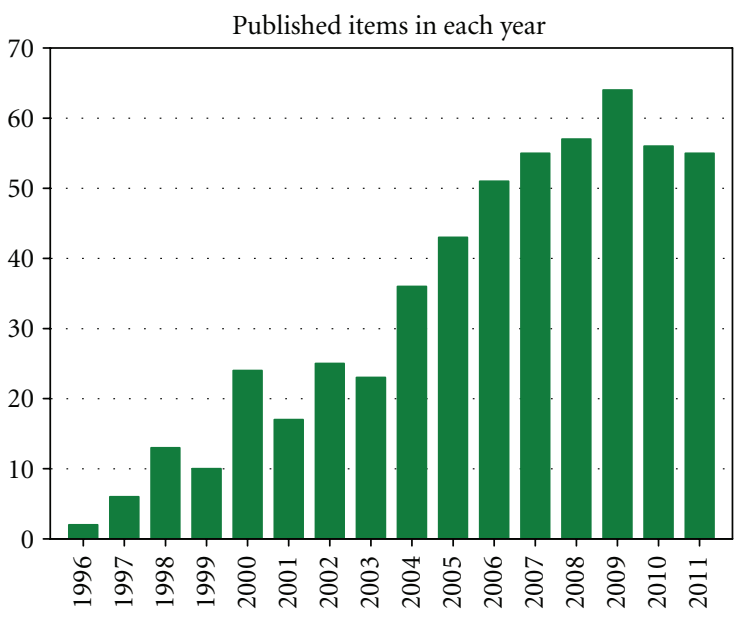

(a)

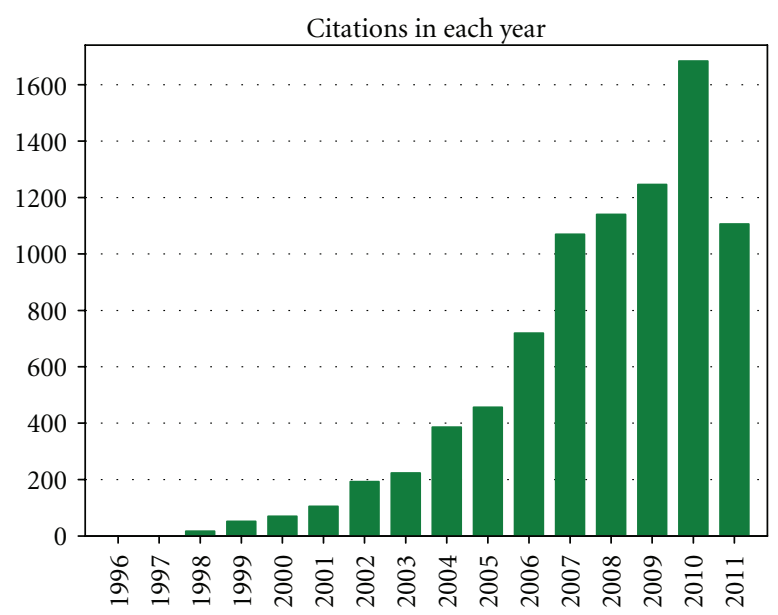

(b)

FIGURE 1: Evolution in the number of papers published (a) and citations received (b) on e-tongues along the last years (consulted: 11 October 2011). Graphs extracted from Thomson Reuters' Web of Science, using keywords "electronic tongue" or "artificial taste."

and optical, if main sensor families need to be quoted. Table 1 sketches the different approaches that can be listed when inspecting the specialized literature. Even, hybrid systems have also been proposed, mainly those combining potentiometric and voltammetric sensors. The combination of electronic noses and electronic tongues to improve detection or identification capabilities in a sensor fusion approach has also been proposed.

As can be seen in Figure 1, the establishment of "electronic tongues" as a consolidated research topic is clear. Near to 500 papers have been already published at the ending of 2011, and growing numbers of citations (ca. 10 000) are accumulated. Every year, new groups contribute to the field, and, specially, the application-type works are increasing. This section will describe the basics of electronic tongue systems, both of qualitative and of quantitative type; it will focus succinctly on different applications developed in our laboratories, employing arrays of electrochemical sensors of the potentiometric and voltammetric families; even, the development of applications employing arrays of biosensors will be described. For a convenient automated operation of the developed devices, some of them have been implemented with the flow injection or sequential injection techniques.
1.1. Data Processing Tools. Let us consider the situation of a sensor array with cross-response features as the departure point. Since all the sensors may respond to all the analytes, a great amount of complex data is generated that must be processed using a multivariable calibration approach. Chemometrics is in charge then, for extraction of the information sought by appropriate processing. This coupling has been declared one of the more clear benefits accounted for in the combination of chemometrics and electrochemical sensors [9]. Depending on the type of application, different methods are available for the processing of data; two of these are ANNs and PCA, as it has already been mentioned. Curiously and as already discussed, if the system employs ANNs, a double biomimicry circumstance occurs, given ANNs are also inspired by the physiology of the animal nervous system. One can classify the different available techniques according to the type of application. If this is a qualitative goal, PCA is the first step as it will either visualize if the samples can be separated in classes (classified) or will identify a specific variety. After this, some pattern recognition or means to predict the membership to any of the classes will be needed; for this, tools like linear discriminant analysis, nearest neighbour, soft independent modelling of 
class analogy (SIMCA), or ANNs can be used. When the purpose is quantitative, different tools are available, given the numeric information is the end result. Some of these are principal component regression (PCR), which departs from a first PCA transformation to build a multivariate regression, partial least squares regression (PLS), or ANNs. When the departure information is extremely complex, a previous feature extraction is needed, which useful to suppress redundant, nonsignificant information and to retain key data. Some examples of procedures used for feature extraction are PCA, fitting of splines or other functions, for example, Legendre polynomials, or Fourier or Wavelet transform.

When one needs to develop a qualitative application with an electronic tongue, the processing of multicomponent data generated by nonspecific sensors is normally performed using principal component analysis (PCA). The aim is to reduce the amount of variables to new latent variables (principal components) in a reduced variable space to facilitate identification or classification; additionally, this new space simplifies the interpretation of the variability contained in the available information. Here, the difficulty is to find an explanation to these principal components according to the sample composition. PCA is a powerful linear unsupervised pattern recognition method that reduces the dimensionality of a multivariate problem and helps to visualize the different categories of multivariate profiles by highlighting similarities and differences between sample clusters. In essence, PCA performs a change of axis directions of the data space in a way to obtain as the first axis those with maximum variance variation. In mathematical terms, $\mathbf{X}$ being the original data matrix, it is recalculated in approximation as the product of two new matrices of reduced dimension $\mathbf{X}=\mathbf{T P}^{\mathrm{T}}$, where $\mathbf{P}$ is the loadings matrix, that is the transformation in the new directions, and $\mathbf{T}$ is the matrix of scores, that is the coordinates in the new directions. Normally, $\mathbf{T}$ and $\mathbf{P}$ are calculated in a way that most of the original variance (ideally more than 90-95\%) is preserved in the first 2-4 directions. In this way, a low dimensional hyperplane is analyzed to examine groupings or trends of $\mathbf{X}$. The calculation of the eigenvector $\left(\mathbf{X}^{\mathrm{T}} \mathbf{X}\right)$ of the loadings permits to rank them in $\%$ of variance explained.

If the quantitative application is the case, let us consider this example, a multidetermination application employing an array of Ion Selective Electrodes (ISEs) as input data. In a rather complex system, where a number of interferents may be present for a given primary ion, a thermodynamical relation can be established using the Nicolsky-Eisenmann expression that defines the response of a sensor (i) towards the activities of the interfering ions, as

$$
E_{i}=K_{i}+s_{i} \log \left[a_{1}+k_{1, j}^{\mathrm{pot}} \cdot\left(a_{j}\right)^{z_{1} / z_{j}}+k_{1, k}^{\mathrm{pot}} \cdot\left(a_{k}\right)^{z_{1} / z_{k}}+\cdots\right],
$$

where $a_{1}$ is the primary ion of this sensor, and $a_{j}, a_{k}$, and so forth the interfering ions of charge $z_{j}$, and $z_{k}, K_{i}$, and $s_{i}$ the electrode constant and sensitivity, respectively. The cross-sensitivity is expressed through the potentiometric selectivity coefficient, $k_{1, h}^{\text {pot }}$, a measure of how an interfering ion $h$ generates a distorting response when measuring the

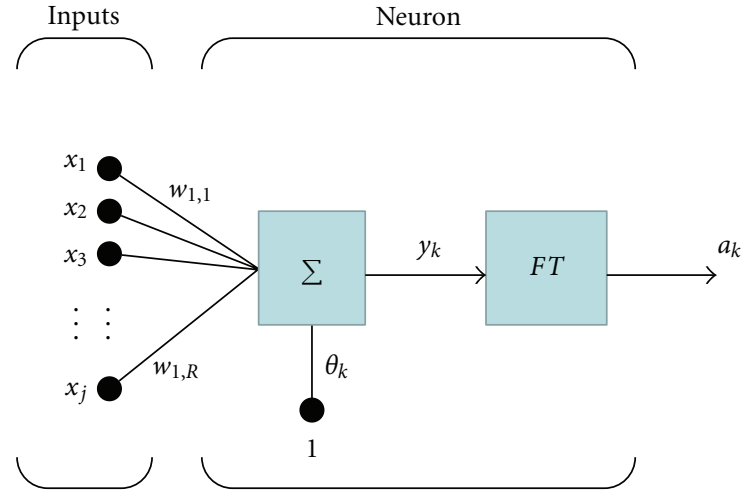

Figure 2: Schematic representation of the perceptron, as data processing element.

species (1). Given that the number of ISEs will be larger than the number of species, for any cross-response situation considering more than two ions, the subsequent equation system that is obtained represents a challenge for any chemometrics specialist.

But instead of the thermodynamical model, various authors propose the use of an ANN for the processing of data obtained through an array of ISEs. Since ANNs are powerful in the modelling of nonlinear systems and the subjacent phenomena are highly nonlinear, preliminary results were very promising, permitting both qualitative and quantitative analysis. Other research groups support the use of PLS regression ahead of ANNs.

The basic processing unit of an ANN (or its building block) is called perceptron, which is a crude approximation to the biological neuron, the cell in the nervous system. It is a decision-making unit with several input connections and a single output, as sketched on Figure 2. A signal $x_{j}$ which is delivered from input $j$ is multiplied on arrival by a connection weight $w_{k, j}$, so that each signal appears at the $k$ perceptron as the weighted value $w_{k, j} \cdot x_{j}$. The perceptron sums the incoming signals and adds a bias $\theta_{k}$ to give a total signal $y_{k}$. To this sum, a transfer function, normally a stepfunction, is applied to produce the output $a_{k}$. Inspired by its physiology, if the sum of inputs is below a threshold value, the neuron is quiescent and remains "off." If the sum reaches the threshold level, the neuron is turned "on" and a message is sent out. Apart from this, the nonlinear processing capability resides in the choice of the transfer function, as when the activation is produced, it can also take a nonlinear profile.

Therefore, the behaviour of the perceptron to certain input information is determined by the weights of its input connections and by the level at which the threshold is set. The transfer function used will also define the shape of the transition step. Knowledge is stored as the values of adjustable parameters $\left(w_{k, j}\right)$, so initially the connection weights are set to small random values. Learning is then the process of adjusting the values in a way that roughly parallels the training of a biological system. A unique condition must be fulfilled: the problem has to be linearly separable, but most significant scientific problems are not. 


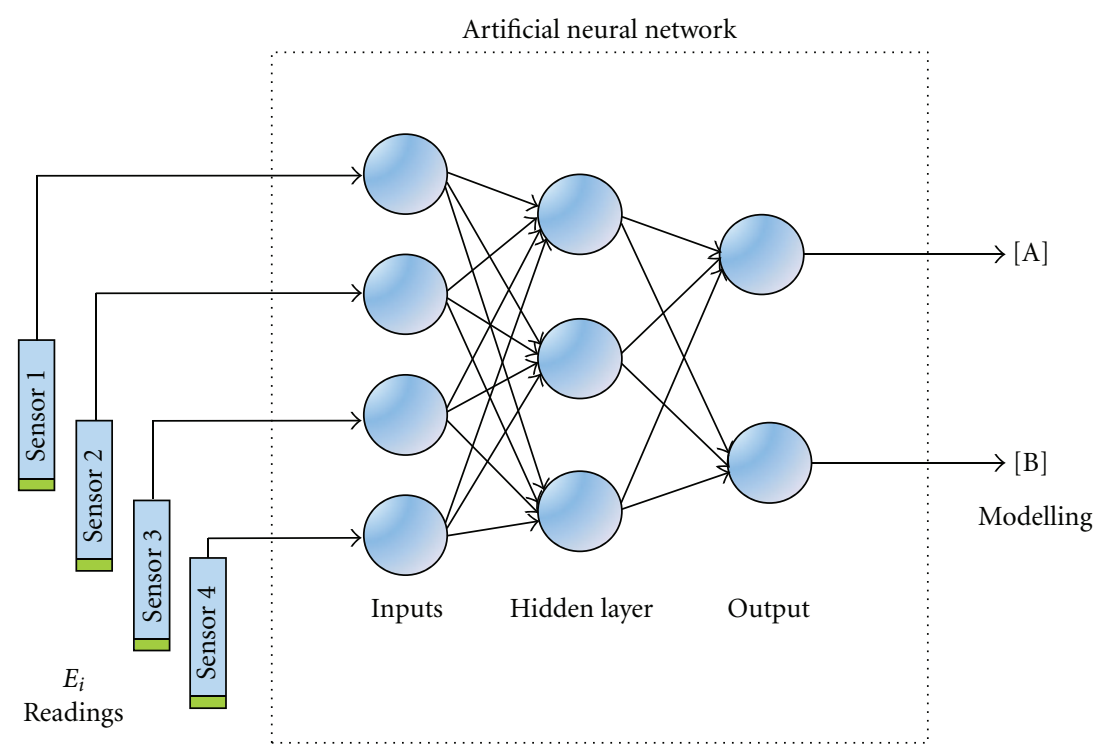

FIGURE 3: The electronic tongue concept applied for multidetermination employing a neural network model.

Putting it into mathematics, the cumulative input is calculated as

$$
y_{k}=\sum w_{k, j} \cdot x_{j}+\theta_{k}
$$

And the firing of the neuron happens if the threshold, as defined by a transference function used, is surpassed:

$$
a_{k}=F T\left\{y_{k}\right\} \text {. }
$$

Although a single perceptron is a limited data processing strategy, a much more powerful approach is the use of sets of associated perceptrons for the final configuration. This can be done in two different ways: first giving the perceptrons neighbours to form a layer of units which share inputs from the environment; secondly by introducing further layers, each taking, as their input, the output from the previous layer. In this way, the network of perceptrons (or artificial neural network, ANN) used for numerical models is known as the multilayer feedforward network and, in its more simple expression, uses a first layer for input information (input layer), a second layer of perceptrons, which are the ones actually computing the model (hidden layer), and a final layer to get results (output layer). An ANN used to process the readings from a sensor array is sketched in Figure 3; the scheme represents the approach for a simultaneous calibration model of two species, A and B, departing from the readings of four ISE sensors. As can be tracked in the figure, departure information (the potential sensor readings, $E_{i}$ ) enters directly into the input layer, whose purpose is just to distribute incoming signals to the next layer; it does not perform any thresholding; thus, these units are not perceptrons in its right sense. Perceptrons in the second layer constitute a hidden entity; as they communicate with the environment only by sending or receiving messages to units in neighbour-connected layers. This hidden layer normally employs one layer of perceptrons for simplicity, but nothing impedes to use more. The output layer provides a link between the artificial network and the outside world, submitting the processed information (here, the sought concentrations). Notice that every perceptron is connected to all units in the adjoining layers, but there are no connections between units in the same layer. That is why it is called a fullyconnected layered feedforward network-messages flow in the forward direction only.

Mathematically, each output represents just a specific linear combination with specific weights from each preceding perceptron, though passage from layer to layer is also modulated by the transfer functions used (usually there is a certain transfer function for all nodes in certain layer):

$$
[X]=F T^{\text {output }}\left\{\theta_{j}^{o}+\sum w_{i, j}^{o} \cdot F T^{\text {hidden }}\left(\sum w_{i, k}^{h} \cdot E_{k}+\theta_{k}^{h}\right)\right\},
$$

where the superscripts o and $\mathrm{h}$ indicate output and hidden layers, respectively, and the input information $\left(E_{k}\right)$ is the different readings from the ISEs. The operation, then, is formed by two stages: first, the ANN response model is built, using some training data, and next the model can be used for prediction of unknown samples.

In this approach, there are many conditions to fix before the training of the ANN can be started. In fact, this is a delicate part of the modelling, as there are too many variants, and experience is the unique way to arrive to a good configuration [10]. First, there is to decide if the input data must suffer any kind of pretreatment. A recommended step here is to normalize the range of the different input channels to avoid any imbalance between them. A second point is to decide the topology of the network; although the number of input and output neurons is fixed by the nature of input and output information, there is no guide as to infer which number of neurons in the hidden layer will yield better ANN models. Concerning architecture of the multilayer network, almost all the recorded chemistry situations employ single hidden layers. To deduce the best 


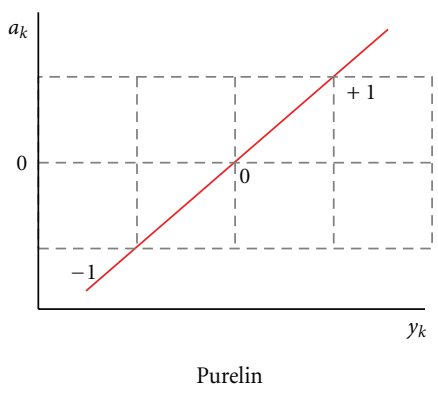

(a)

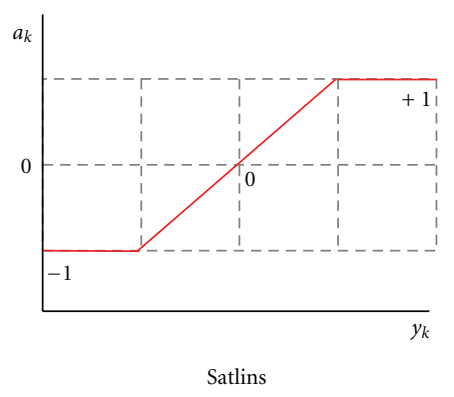

(b)

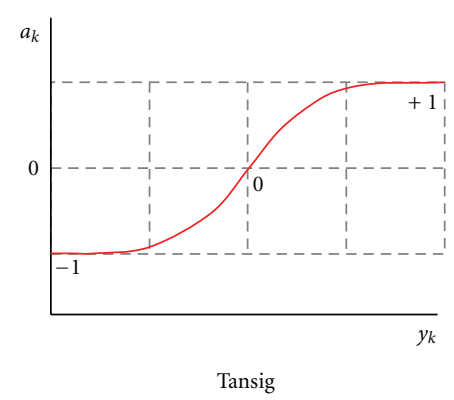

(c)

FiguRE 4: Representation of three commonly used transfer functions purelin, satlins, and tansig.

number of neurons, the recommendation is trial and error, beginning with a large number and decreasing it until the model gets worse. In this way, like when fitting polynomials, some degree of adjustment is firstly obtained, subsequently improved reducing the number of neurons, and once it deteriorates due to a manifestly simple network, the optimal configuration is met. Another factor is the kind of transfer functions used, normally the same for the whole layer. The input layer, playing only a distributive, role uses a linear transfer function, called purelin, while the hidden and output layers can use one among around a dozen possible functions. Some of the transfer functions that can be used are shown on Figure 4. It is common to use sigmoidal transfer functions in the hidden layer, like the log-sigmoidal (logsig) and the tan-sigmoidal (tansig). The logsig function generates outputs between 0 and 1 , at which the sum of the outputs goes toward infinity. The tansig-function is very similar to the logsig, but it generates outputs between -1 and 1. A saturatedlinear function (satlins) represents a linear correspondence but with a way to avoid saturation at its output. Which transfer function will be best for our problem will depend on the nature of the relationship considered, and again, different possibilities have to be checked for a given case. Other factors to consider are how to accomplish the "learning" of the network, how to check the progress of this learning process, and how to avoid some vices that can arise during learning.

If we consider now the process of learning, in the elementary perceptron, the learning rules are unambiguous: for a set of known samples, the weights $w_{k, j}$ are adjusted until the obtained outputs agree with the expected values. Matters are more complicated in a network, because it must be established how changes in the connection weights should be allocated to connections between different layers to promote learning.

A common solution to this problem is backpropagation. Let us represent as $o_{p k}$ the output of perceptron $k$ for a sample $p$ in the training set, calculated with an expression like (5); analogously, let us represent as $t_{p k}$ the target value. The error signal $\left(t_{p k}-o_{p k}\right)$ is calculated, and a proportion of the error signal is allocated to the various connections in the network (backpropagated), tuning the values of connection weights $\left(w_{k j}\right)$. The goal of this procedure is to reduce the error signal. Using standard backpropagation, the error signals are collected for all output units and all training targets, and the connection weights are adjusted at the end of every epoch, that is, after all samples in the training set have been shown to the network once.

Backpropagation therefore adjusts the weights to minimize the error function $F_{p}$, defined as the semisum of the individual output unit errors for all samples $(k)$ in the training set:

$$
F_{p}=\frac{1}{2} \sum_{k}\left(t_{p k}-o_{p k}\right)^{2}
$$

In this way, the learning process of an ANN is equivalent to a minimization in a multidimensional space (the space of connection weights). A means to accomplish this is to use the gradient-descent algorithm, an iterative optimisation procedure in which the connection weights are adjusted in a fashion which reduces the error most rapidly, by moving the system downwards in the direction of maximum gradient. The weight of a connection at stage $(t+1)$ of the training is related to its weight at stage $(t)$ by $(6)$ :

$$
w_{k j}(t+1)=w_{k j}(t)-\eta \frac{\partial F_{p}}{\partial w_{k j}},
$$

where $\eta$ is a gain term, known as the training rate factor; next, it is reexpressed as

$$
w_{k j}(t+1)=w_{k j}(t)+\eta \delta_{p k} \cdot o_{p k},
$$

where $\delta$ is the size of change; the product $\delta_{p k} \cdot o_{p k}$ represents the gradient contribution. The training rate factor varies between 0 and 1 and accelerates or slows down the descent towards the global minimum of the system; numbers around 0.5 are of typical use here. It is possible to derive expressions prescribing the size of the changes that must be made at the connection weights to reduce the error signal.

For the output layer,

$$
\delta_{p k}=k o_{p k}\left(1-o_{p k}\right)\left(t_{p k}-o_{p k}\right) .
$$

For the hidden layer,

$$
\delta_{p k}=k o_{p k}\left(1-o_{p k}\right) \sum_{i} \delta_{p i} w_{k i} .
$$




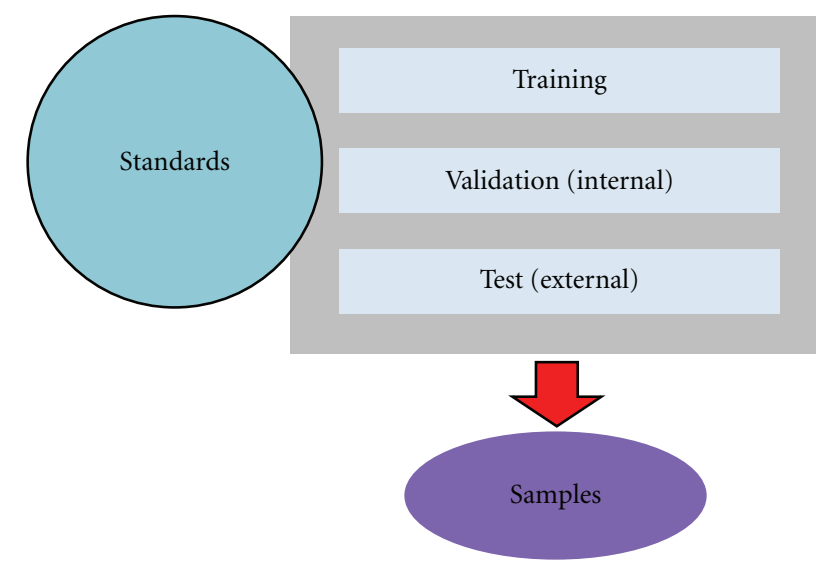

FIGURE 5: Subdivision of the sample set in the different subsets recommended to prepare the training process of an ANN model.

These expressions, which are known as the generalized delta rule, show that the extent of the adjustment of connection weights to hidden layers depends upon errors in the subsequent layers, so modifications are made first to the output layer weights, and then the error is propagated successively back through the hidden layers-this is referred to as backpropagation (of error). Each unit receives an amount of the error signal which is in proportion to its contribution to the output signal, and the connection weights are adjusted by an amount proportional to this error.

Backpropagation by gradient descent is generally a reliable procedure; nevertheless, it has its limitations: it is not a fast training method, and it can be trapped in local minima. To avoid the latter, a variant of the above algorithm called gradient-descent with momentum (GDM) introduces a third term, $\beta$, referred to as the momentum:

$$
w_{k j}(t+1)=w_{k j}(t)+\eta \delta_{p k} o_{p k}+\beta \Delta w_{k j}(t) .
$$

$\beta$ takes a fixed value between 0 and 1 and serves to reduce to the probability of the system being trapped in a local minimum.

One of the problems that may occur during neural network training is called overfitting. This situation occurs when the error on the training set is driven to a very small value, but the new data presented to the network shows a large error. The overfitting concept means that the network has memorized the training examples, but the generalization to new situations is incorrect. In practice, there are two methods that can be used to avoid overfitting, Bayesian regularization, and Early stopping. In the first case, the idea is to select the simplest network possible, and this is simplified by eliminating nodes whose weight connections are not significant enough. In the second case, three subsets of data are employed, see Figure 5. A first subset is used for training the model, a second (validation set) is used to check if overfitting is taking place (detected as an increase in the validation error), and a third set (the external test set) is used only to compare performance between different models.

All these precautions represent an increased experimental effort in generating data for the numerical model, which can be one of the drawbacks when working with ANNs. In order to achieve convergence with a proper modelling ability and a demanded level of accuracy and precision, two independent data sets are used at least, a training set and an (internal) test or validation set, although the third set is also suggested. Each set contains two kinds of information that interrelate. The first type is formed by the responses of the sensor array (patterns); the second is their corresponding searched information (targets), in a quantitative application case, the concentration values of the analytes. This training set must be large enough and contain sufficient variability to yield a proper modelling of the response. In order to assure the quality of the final results, some system validation is required. For this purpose, the second set of data, the validation set, is used. From the total of generated cases, one can equally distribute the available cases between the different subsets or reserve the largest part of the data (up to $50 \%)$ for training. Reference authors in the field recommend the use of 5-10 training samples per connection weight in the ANN model, which can reach hundreds or thousands of cases for complex network structures. Ways to generate the complete set is through experimental design schemes, for example, a factorial design or others. When working with electronic tongues employing ISEs, two other situations are also typical, the use of a collection of sample varieties (juices, wines, an intermediate industrial product, etc.) that need to bring some reference value (target, e.g., a parallel analytical determination) in order to build the training set, or a set of examples generated by accumulated microadditions of a certain standard in a predefined background.

\section{Electronic Tongues Employing Potentiometric Sensors}

Qualitative applications will be shown departing from an array of eight PVC-membrane potentiometric sensors, classifying food varieties and predicting properties of fruit juices, in combination with principal component analysis (PCA) [11]. The first attempt was to classify synthetic samples prepared with controlled variability. Once this ability is proven, satisfactory classification results are presented for commercial waters, orange-based drinks, and tea samples. An interesting correlation is achieved between the natural juice content and its first calculated component, which allows for a very simple tool for screening purposes.

A similar array of potentiometric sensors, this time with devices sensitive to alkaline ions, can be used for the monitoring of ammonium without the removal of sodium and potassium interfering ions [12]. The case involved the use of a nonactin-based potentiometric membrane, plus sensors for sodium and potassium, as well as some of generic response to the alkaline family. The described case is of environmental relevance as it may form the base for the unattended determination of ammonium pollutant in surface waters [13]. An array of eight nonspecific potentiometric sensors with PVC membranes was used in combination with multivariate calibration for the simultaneous determination of the alkaline species. Their exact formulation is presented in Table 2. Signals were processed by using a multilayer 
TABLE 2: Formulation of the ion selective membranes employed in the construction of the potentiometric sensor array.

\begin{tabular}{lccc}
\hline Sensor & PVC (\%) & Plasticizer (\%) & Recognition element (\%) \\
\hline $\mathrm{NH}_{4}{ }^{+}$ & 33 & Butylpentyladipate (66) & Nonactin (1) \\
$\mathrm{K}^{+}$ & 30 & Dioctylsebacate (66) & Valinomycin $(3)^{\mathrm{a}}$ \\
$\mathrm{Na}^{+}$ & 22 & Nitrophenyloctylether (70) & Bis [(12-crown-4) methyl]-2-dodecyl-2-methylmalonate (6) \\
$\mathrm{H}^{+}$ & 33 & Dioctylsebacate (66) & Tridodecylamine (1) \\
$\mathrm{Cl}^{-}$ & 30 & Nitrophenyloctylether (65) & Tetradodecylammonium chloride (5) \\
$\mathrm{NO}_{3}{ }^{-}$ & 30 & Dibutylphtalate (67) & Tetraoctylammonium nitrate (3) \\
Generic 1 $_{\text {Generic 2 }}^{29}$ & 29 & Dioctylsebacate (67) & Dibenzo (18-crown-6) (4) \\
\hline
\end{tabular}

${ }^{\mathrm{a}}$ The formulation includes potassium tetrakis(4-chlorophenyl)borate as an additive.

artificial neural network (ANN). The ANN configuration used was optimized by using 8 neurons in the input layer, 5 in the hidden layer and 3 in the output layer. Use of the Bayesian regularization algorithm allowed the rapid building of an accurate model, as confirmed by random multistarting of network weights. The system was used to analyze synthetic and river water, waste water, and fertilizer samples. Correct results were obtained for the three ions in synthetic and real water samples; in fertilizers, ammonium ion can be determined, while sodium and potassium show biased results.

A similar case was derived from the latter, which is the monitoring of nutrients in recirculated feed solutions of greenhouse facilities [14]. There, the need was to monitor nutrients like ammonium, potassium, and nitrate and, simultaneously, detect the presence of undesired species like sodium or chloride, which accumulate during long-time operation. The case for cations was attempted with sensors sensitive to alkaline ions plus a generic response membrane; the anions were resolved employing PVC membrane sensors for nitrate and chloride and a generic response to anions unit. Temperature dependence was also counterbalanced through measurement and modelling of its effect. The response model was built employing ANNs and samples prepared from a factorial experimental design. A further evolution of the system for the determination of ammonium consisted of the conversion of ammonium sensors to urea biosensors through covalent immobilization of urease. With this array, which incorporated urea biosensors plus ammonium and alkaline-ion sensors, it was possible to determine urea in clinical samples without the need to separate endogenous ammonium or interfering sodium and potassium ions [15].

2.1. Use of Flow Systems. In the work with electronic tongues, soon we realized the large experimental efforts related with the generation of information to fed the chemometric tools. In the qualitative application, the workload is the introduction and measurement of a sufficiently large set of samples representing the different possible cases. For a multidetermination case, it stands on the preparation of a large set of standards, with different proportions of the considered substances, a huge task if the number of species is large. In both cases, the use of flow systems may help in the operation of electronic tongues [16], especially to facilitate the processing of large sets of samples. This was first demonstrated with a FIA system employing different flow-through

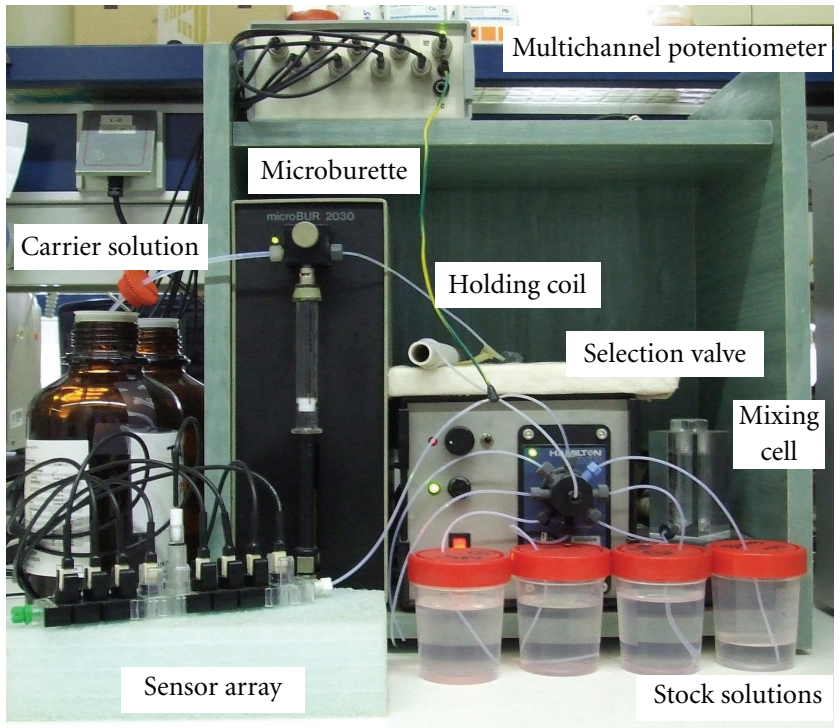

Figure 6: Image of the sequential injection analysis system employed to develop automated electronic tongues.

sensors for nitrate and chloride that demonstrated its utility in removing the chloride interference for the determination of nitrate [17]. The use of large sets of samples is also forced by ANNs, since a larger number of samples than the number of interconnections is recommended to build the model [10]. For this reason, we developed an automated system employing the sequential injection analysis technique, capable of preparing arbitrary mixtures of samples from more concentrated stock solutions [18]. With this system, sketched in Figure 6, it is possible to generate automatically ca. 100 samples and obtain their measurements. Different applications will be described, such as the combined determination of alkaline ions [19] or the combined determination of alkaline-earth ions [20], each from an array of 5 tubular flowthrough PVC-membrane electrodes.

In this case, the simultaneous determination of $\mathrm{Mg}^{2+}$, $\mathrm{Ca}^{2+}$, and $\mathrm{Ba}^{2+}$ in water was done using a PVC-membrane potentiometric sensor array and multivariate calibration. The response model was based on the use of a multilayer artificial neural network (ANN). The information needed for training or generation of the model was obtained with the aid of an automated analytical system based on the 
sequential injection analysis (SIA) technique. The modelling ability was verified with an external set of standards, and then the determinations were performed in real samples of mineral waters, where close results for $\mathrm{Mg}^{2+}$ and $\mathrm{Ca}^{2+}$ were obtained to those obtained with reference methods. The determination of $\mathrm{Ba}^{2+}$ can be considered as semiquantitative for synthetic samples-due to the absence of $\mathrm{Ba}^{2+}$ in mineral waters, its concentration in real samples was not measured.

More recently, the use of the automated system has permitted us to adapt our procedures to record the transient response corresponding to a sample step [21]. The novelty is the use of the dynamic components of the signal in order to better discriminate or differentiate a sample. An electronic tongue based on the transient response of an array of non-specific-response potentiometric sensors was thus developed. As before, the SIA system was used in order to automate its training and operation. The use of the transient recording entails the dynamic nature of the sensor's response, which can be of high information content, of primary ions, and also of interfering ions; these may be better discriminated if the kinetic resolution is added. In the study, significant information contained in the transient response of each sensor from a sensor array formed by five all-solidstate potentiometric sensors was extracted carefully. The tool employed for this purpose was the Fourier transform, from which a number of coefficients were fed into an artificial neural network (ANN) model, used to perform a quantitative multidetermination. The studied case was the analysis of mixtures of $\mathrm{Ca}^{2+}, \mathrm{Na}^{+}$, and $\mathrm{K}^{+}$. Obtained performance was compared with the more traditional automated electronic tongue using final steady-state potentials, showing an improved modelling capability. A similar study was performed for anions, where nitrate, chloride, and hydrogen carbonate were simultaneously determined from an array of ISEs for anions [22].

\section{Electronic Tongues Employing Voltammetric Sensors}

Some examples will also be described with the use of voltammetric sensors, for example in the classification of foods or beverages, or quantification of mixtures of oxidizing substances. Differently to the potentiometric case, the voltammetric signal is inherently linear, although its dimension is greater. That is, different oxidizable compounds present in a sample contribute in a certain proportion to the current signal, although a vector of currents is generated per each electrode used in the array:

$$
i_{k}(V)=i_{o}+k_{1} \cdot C_{1}+k_{2} \cdot C_{2}+k_{3} \cdot C_{3}+\cdots,
$$

where $i_{k}(V)$ is the vector of currents of electrode $k$ and the concentrations of the different oxidizable compounds present in a sample are denoted by $C_{1}, C_{2}, C_{3}$, and so forth.

One important fact associated with voltammetric electronic tongues is the higher dimensionality that characterizes them, making the data treatment process much more difficult. Normally, each voltammogram may be formed by several dozen, even hundreds of current intensities; although one voltammogram may be easily processed by the algorithms intrinsically doing data reduction, such as PCA or PLS, this is not the case if ANNs are used. Also, the use of several electrodes in the array, providing a vector measure per each sample, also complicates the situation. The three-way matrix which is generated (samples $\times$ currents $\times$ sensors) is then too complex for the ordinary data treatment procedures. Approaches to cope with this are the unfolding, for example, combining of the sensor signals into one single vector, although its excessive length or the introduced discontinuities may be a problem. Other approaches used are to compress the data to some reduced, compressed values, that may represent the original measure and allow the application of, for example, ANNs. This has been accomplished by performing feature extraction from the original signals, if the measured signals have clear shapes or profiles to interpret, or, in a more generic way, by reducing complexity through data compression strategies, like PCA, Fourier, or Wavelet transforms. And, finally, there are the multiway treatment procedures, like PARAFAC or N-PLS, capable of treating the three-way data matrix directly, very rarely employed up to this moment with electronic tongues, probably due to the high complexity involved.

A new approach towards a voltammetric electronic tongue was developed in our laboratories [23]. Automation of the system was achieved with the use of an SIA system. Design and construction of a small detection device containing 3 working electrodes was carried out. Platinum, gold, and epoxy-graphite discs were used for this purpose. $\mathrm{An} \mathrm{Ag} / \mathrm{AgCl}$ reference electrode was integrated into the measuring cell in order to minimise electrical noise. Three oxidizable compounds of clinical interest, that is, ascorbic acid, uric acid, and paracetamol, could be quantified by the system. Employing the voltammograms as departure information, artificial neural networks (ANN) have been used as chemometric tool for the modelling of the system (see Figure 7). The three voltammograms were added up, summing the currents for each polarization potential, and simplifying the original data to a simple vector, that were then easily processable by ANNs. An interesting similar work employing a FIA system was developed for environmental application, permitting a quick estimation of the chemical oxygen demand [24].

The use of arrays of voltammetric sensors has recently enabled an application very much desirable from the industrial sector, which is the use of electronic tongue systems to monitor and to detect defects during production of wine [5]. In our case, we have proposed the use of an array of 6 voltammetric electrodes modified with different nanocomponents or conducting polymers, in order to generate a sufficiently differentiated response [25]. Table 2 summarizes the composition of the sensors used. The system has been successfully applied in the identification of wine classes and in the identification of some types of spoilage. In a second study, the same sensor array could be used to differentiate between different fabrication variants of the catalan cava sparkling wine, in what is known "brut," "brut-nature," "dry," "medium-dry," or "sweet" [26], an application that almost rivals sommeliers in cava tasting. In 

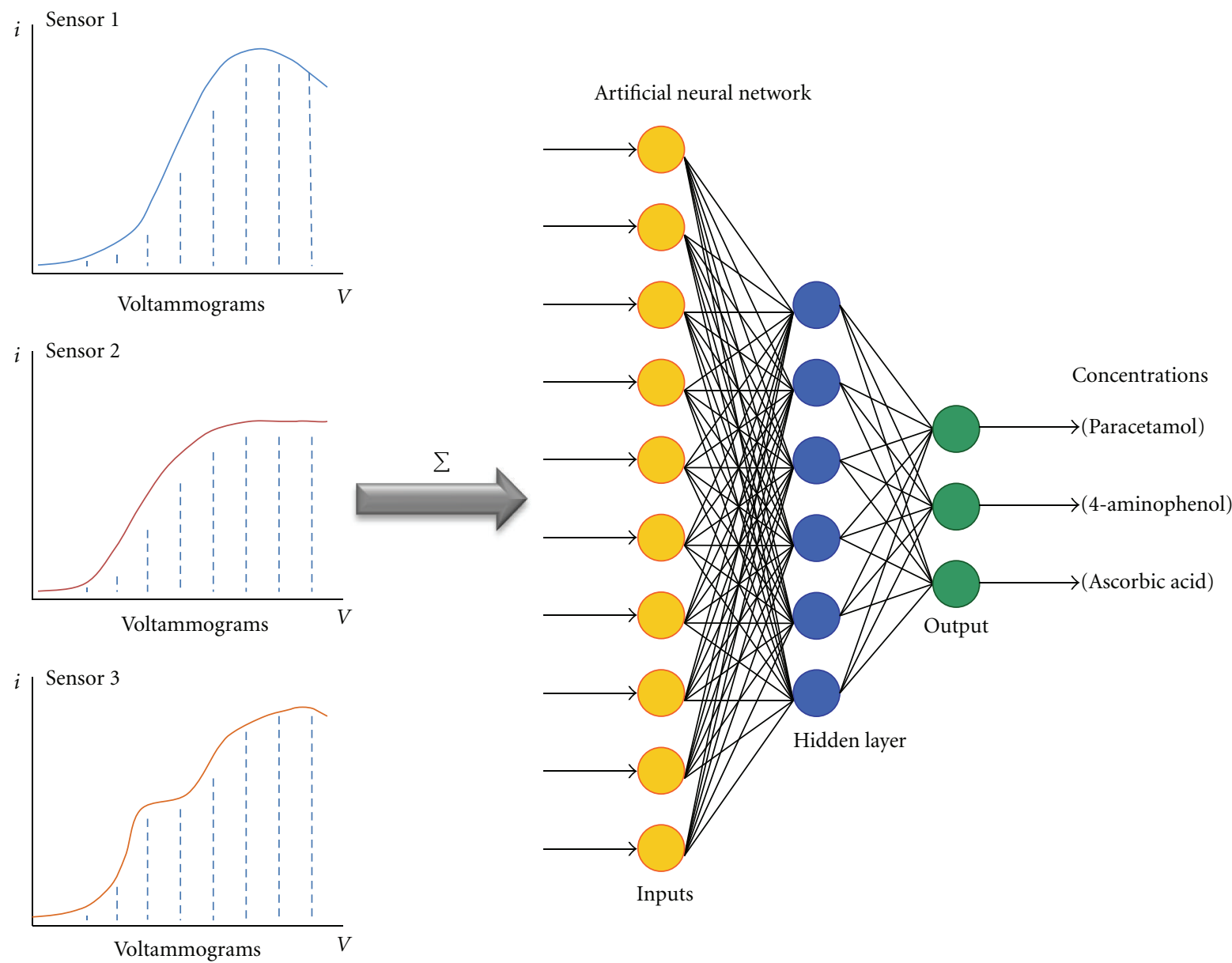

FIGURE 7: Concepts in a voltammetric electronic tongue performing a quantitative application.

TABLE 3: Composition of the voltammetric sensors included in the array.

\begin{tabular}{lccc}
\hline Sensor & \% graphite & Modifier (\%) & $\%$ epoxy \\
\hline GEC & 50 & - & 50 \\
PC & 48 & Cobalt phthalocyanine (2) & 50 \\
Pt & 48 & Platinum nanoparticles (2) & 50 \\
Cu & 48 & Copper nanoparticles (2) & 50 \\
PPY & 48 & Polypyrrole (2) & 50 \\
PANI & 48 & Polyaniline (emeraldine salt) (2) & 50 \\
\hline
\end{tabular}

both cases, the data processing involved was the unfolding of voltammograms and the qualitative transformation PCA (Table 3).

\section{Electronic Tongues Employing Biosensors}

The biosensor array for the determination of urea [15] has already been described, as it was formed essentially by potentiometric sensors. As an example of a voltammetric electronic tongue employing biosensors, the determination of different phenolic compounds from the overlapped spectra obtained from a tyrosinase enzyme biosensor is an important example. Determination of phenols is significant given their toxicity, even at very low concentration levels. Amperometric determination of phenols is a simple technique available. Direct oxidation of phenols can be used, but another possibility is the use of polyphenol oxidase (tyrosinase) enzyme biosensors that oxidises the phenolic compounds into their corresponding quinones. Reduction of the resulting quinones accomplishes the amplification of the amperometric signal, as long as the result of the reduction process is the corresponding cathecol, this being able to be oxidised again by the polyphenol oxidase immobilized on the surface of the biosensor. In this communication, simultaneous determination of different phenols was carried out combining biosensor measurements with chemometric tools, in what is known as electronic tongue. The departure information used was the overlapped reduction voltammogram generated with the amperometric biosensor based on polyphenol oxidase. ANNs were again used for extraction and quantification of each compound. Phenol, cathecol, and $m$-cresol formed the three-analyte study case resolved in this work. Good prediction ability was attained; hence, the separate quantification of these three phenols was accomplished [27]. The proposal of electronic tongues has also been extended to the use of inhibition-based biosensors, such as those employed for the determination of pesticides [28]; the principle, here, is to use enzymes from different 
biological origins, that may show different inhibitory degree to a set of substances. The use of biosensors in the sensor array has coined the term bioelectronic tongue $[1,5]$.

\section{Conclusions}

The growing demand for (bio)chemical information in the different economic sectors or in the environment and the public concern on health and quality are clearly driving for new trends in analytical methodologies. Electrochemical (bio)sensors are a clear option for a fast, simple, and cheap gathering of information, although sensors alone cannot solve all the existing situations. In the last decade, a new trend in the sensor field has appeared which is to couple multidimensional sensor information with advanced computer processing strategies; this approach, known as electronic tongue, can take profit of electrochemical sensors and biosensors from the potentiometric and voltammetric families, among others [29]. In perspective, electronic tongues can be one of the clearest benefits of the application of chemometrics in (bio)sensor research. One of the clearest fields of application for electronic tongues can be the food field, where the automated sensory control of fabrication batches and detection of production defects can be an irreplaceable contribution, 24/7. Examining the progress trend, the field seems to be now mature, and perhaps a more complete commercial dissemination is the only event still to observe. Some research lines that seem to be active for the next years are the increase in complexity of new electronic tongues, with higher number of sensors, or with more complex information generated, for example, incorporating the dynamic dimension or other additional dimensions in the signal. Therefore, the multiway processing in electronic tongues will be a must. Also, the use of hybrid electronic tongues, joining in their array sensors from different nature, and in demand for special data treatment approaches too, in this case that may be named data fusion. Finally, the irruption of electronic tongues into the biotechnological field seems a natural trail of progress, where the progressive incorporation of biosensors of different kinds in the operation of electronic tongues is already an initiated trend.

\section{Acknowledgments}

All the different works described would not have been possible without the contribution of past and current Ph.D. students at the Sensors and Biosensors Group: Jordi Gallardo, Albert Gutés, Montserrat Cortina, Daniel Calvo, Michael Grössl, Christina Ecker, Manuel Gutiérrez, Juan Manuel Gutiérrez, Aitor Mimendia, Raul Cartas, Deivy Wilson, and Xavier Cetó. This work is being supported by Spanish Ministry of Science and Innovation, Project CTQ2010-17099, and by Program ICREA Academia.

\section{References}

[1] M. del Valle, "Electronic tongues employing electrochemical sensors," Electroanalysis, vol. 22, no. 14, pp. 1539-1555, 2010.
[2] P. Ciosek and W. Wroblewski, "Sensor arrays for liquid sensing-electronic tongue systems," Analyst, vol. 132, no. 10, pp. 963-978, 2007.

[3] L. Escuder-Gilabert and M. Peris, "Review: highlights in recent applications of electronic tongues in food analysis," Analytica Chimica Acta, vol. 665, no. 1, pp. 15-25, 2010.

[4] A. Riul Jr., C. A. R. Dantas, C. M. Miyazaki, and O. N. Oliveira, "Recent advances in electronic tongues," Analyst, vol. 135, no. 10, pp. 2481-2495, 2010.

[5] J. Zeravik, A. Hlavacek, K. Lacina, and P. Skladal, "State of the art in the field of electronic and bioelectronic tonguestowards the analysis of wines," Electroanalysis, vol. 21, no. 23, pp. 2509-2520, 2009.

[6] M. del Valle, "Potentiometric electronic tongues applied in ion multidetermination," in Electrochemical Sensor Analysis, S. Alegret and A. Merkoçi, Eds., pp. 721-753, Elsevier, Amsterdam, The Netherlands, 2007.

[7] M. Holmberg, M. Eriksson, C. Krantz-Rülcker et al., "2nd workshop of the second network on artificial olfactory sensing (NOSE II)," Sensors and Actuators, B, vol. 101, no. 1-2, pp. 213-223, 2004.

[8] Y. Vlasov, A. Legin, A. Rudnitskaya, C. Di Natale, and A. D’Amico, "Nonspecific sensor arrays ("electronic tongue") for chemical analysis of liquids: (IUPAC technical report)," Pure and Applied Chemistry, vol. 77, no. 11, pp. 1965-1983, 2005.

[9] B. K. Lavine and J. Workman, "Chemometrics," Analytical Chemistry, vol. 74, no. 12, pp. 2763-2769, 2002.

[10] F. Despagne, "Neural networks in multivariate calibration," Analyst, vol. 123, no. 11, pp. R157-R178, 1998.

[11] J. Gallardo, S. Alegret, and M. del Valle, "Application of a potentiometric electronic tongue as a classification tool in food analysis," Talanta, vol. 66, no. 5, pp. 1303-1309, 2005.

[12] J. Gallardo, S. Alegret, R. Muñoz et al., "An electronic tongue using potentiometric all-solid-state PVC-membrane sensors for the simultaneous quantification of ammonium and potassium ions in water," Analytical and Bioanalytical Chemistry, vol. 377, no. 2, pp. 248-256, 2003.

[13] J. Gallardo, S. Alegret, R. Munoz, L. Leija, P. R. Hernandez, and M. del Valle, "Use of an electronic tongue based on all-solidstate potentiometric sensors for the quantitation of alkaline ions," Electroanalysis, vol. 17, no. 4, pp. 348-355, 2005.

[14] M. Gutierrez, S. Alegret, R. Caceres, J. Casadesús, O. Marfà, and M. del Valle, "Application of a potentiometric electronic tongue to fertigation strategy in greenhouse cultivation," Computers and Electronics in Agriculture, vol. 57, no. 1, pp. 1222, 2007.

[15] M. Gutierrez, S. Alegret, and M. del Valle, "Potentiometric bioelectronic tongue for the analysis of urea and alkaline ions in clinical samples," Biosensors and Bioelectronics, vol. 22, no. 9-10, pp. 2171-2178, 2007.

[16] A. Gutés, F. Céspedes, and M. del Valle, "Electronic tongues in flow analysis," Analytica Chimica Acta, vol. 600, no. 1-2, pp. 90-96, 2007.

[17] J. Gallardo, S. Alegret, and M. del Valle, "A flow-injection electronic tongue based on potentiometric sensors for the determination of nitrate in the presence of chloride," Sensors and Actuators, B, vol. 101, no. 1-2, pp. 72-80, 2004.

[18] A. Gutes, F. Cespedes, S. Alegret, and M. del Valle, "Sequential injection system with higher dimensional electrochemical sensor signals: part 1. Voltammetric e-tongue for the determination of oxidizable compounds," Talanta, vol. 66, no. 5, pp. 1187-1196, 2005.

[19] M. Cortina, A. Gutes, S. Alegret, and M. del Valle, "Sequential injection system with higher dimensional electrochemical 
sensor signals: part 2. Potentiometric e-tongue for the determination of alkaline ions," Talanta, vol. 66, no. 5, pp. 11971206, 2005.

[20] D. Calvo, M. Größl, M. Cortina, and M. del Valle, "Automated SIA system using an array of potentiometric sensors for determining alkaline-earth ions in water," Electroanalysis, vol. 19, no. 6, pp. 644-651, 2007.

[21] D. Calvo, A. Duran, and M. del Valle, "Use of sequential injection analysis to construct an electronic-tongue. Application to multidetermination employing the transient response of a potentiometric sensor array," Analytica Chimica Acta, vol. 600, no. 1-2, pp. 97-104, 2007.

[22] M. Cortina, A. Duran, S. Alegret, and M. del Valle, "A sequential injection electronic tongue employing the transient response from potentiometric sensors for anion multidetermination," Analytical and Bioanalytical Chemistry, vol. 385, no. 7, pp. 1186-1194, 2006.

[23] A. Gutes, D. Calvo, F. Cespedes, and M. del Valle, "Automatic sequential injection analysis electronic tongue with integrated reference electrode for the determination of ascorbic acid, uric acid and paracetamol," Microchimica Acta, vol. 157, no. 1-2, pp. 1-6, 2007.

[24] A. Gutes, F. Cespedes, M. del Valle, D. Louthander, C. KrantzRülcker, and F. Winquist, "A flow injection voltammetric electronic tongue applied to paper mill industrial waters," Sensors and Actuators, B, vol. 115, no. 1, pp. 390-395, 2006.

[25] J. M. Gutierrez, L. Moreno-Baron, M. I. Pividori, S. Alegret, and M. del Valle, "A voltammetric electronic tongue made of modified epoxy-graphite electrodes for the qualitative analysis of wine," Microchimica Acta, vol. 169, no. 3, pp. 261-268, 2010.

[26] X. Ceto, J. M. Gutierrez, L. Moreno-Baron, S. Alegret, and M. del Valle, "Voltammetric electronic tongue in the analysis of cava wines," Electroanalysis, vol. 23, no. 1, pp. 72-78, 2011.

[27] A. Gutes, A. B. Ibañez, F. Cespedes, S. Alegret, and M. del Valle, "Simultaneous determination of phenolic compounds by means of an automated voltammetric "electronic tongue"," Analytical and Bioanalytical Chemistry, vol. 382, no. 2, pp. 471-476, 2005.

[28] G. Valdes-Ramírez, M. Gutierrez, M. del Valle, M. T. RamírezSilva, D. Fournier, and J. L. Marty, "Automated resolution of dichlorvos and methylparaoxon pesticide mixtures employing a Flow Injection system with an inhibition electronic tongue," Biosensors and Bioelectronics, vol. 24, no. 5, pp. 1103-1108, 2009.

[29] M. del Valle, "Electrochemical micro(bio)sensor arrays," Microchimica Acta, vol. 163, no. 1-2, pp. 1-2, 2008.

[30] Y. Vlasov, A. Legin, and A. Rudnitskaya, "Cross-sensitivity evaluation of chemical sensors for electronic tongue: determination of heavy metal ions," Sensors and Actuators, B, vol. 44, no. 1-3, pp. 532-537, 1997.

[31] L. Moreno, A. Merlos, N. Abramova, C. Jimenez, and A. Bratov, "Multi-sensor array used as an "electronic tongue" for mineral water analysis," Sensors and Actuators, B, vol. 116, no. 1-2, pp. 130-134, 2006.

[32] C. Krantz-Rülcker, M. Stenberg, F. Winquist, and I. Lundström, "Electronic tongues for environmental monitoring based on sensor arrays and pattern recognition: a review," Analytica Chimica Acta, vol. 426, no. 2, pp. 217-226, 2001.

[33] M. Ferreira, A. Riul Jr., K. Wohnrath, F. J. Fonseca, O. N. Oliveira Jr., and L. H. C. Mattoso, "High-performance taste sensor made from Langmuir-Blodgett films of conducting polymers and a ruthenium complex," Analytical Chemistry, vol. 75, no. 4, pp. 953-955, 2003.
[34] J. J. Lavigne, S. Savoy, M. B. Clevenger et al., "Solutionbased analysis of multiple analytes by a sensor array: toward the development of an "electronic tongue"," Journal of the American Chemical Society, vol. 120, no. 25, pp. 6429-6430, 1998.

[35] G. Sehra, M. Cole, and J. W. Gardner, "Miniature taste sensing system based on dual SH-SAW sensor device: an electronic tongue," Sensors and Actuators, B, vol. 103, no. 1-2, pp. 233239, 2004. 


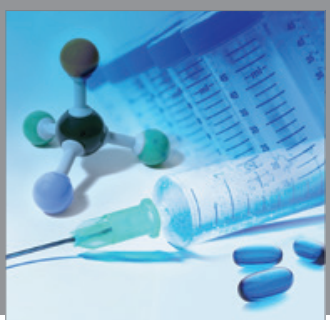

International Journal of

Medicinal Chemistry

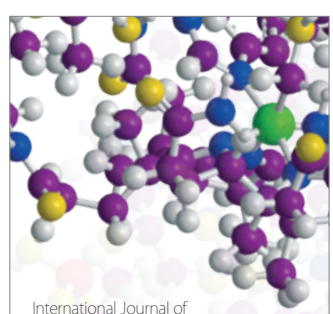

Carbohydrate Chemistry

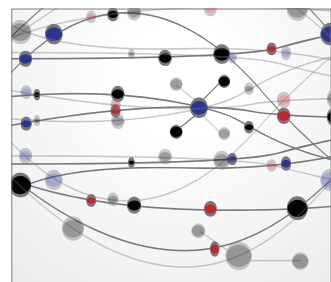

The Scientific World Journal
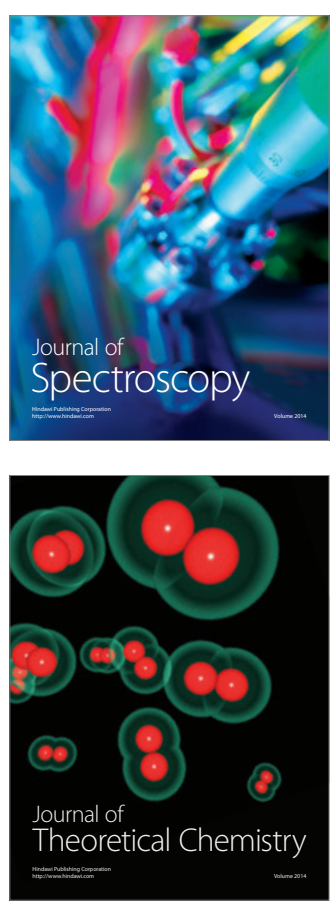
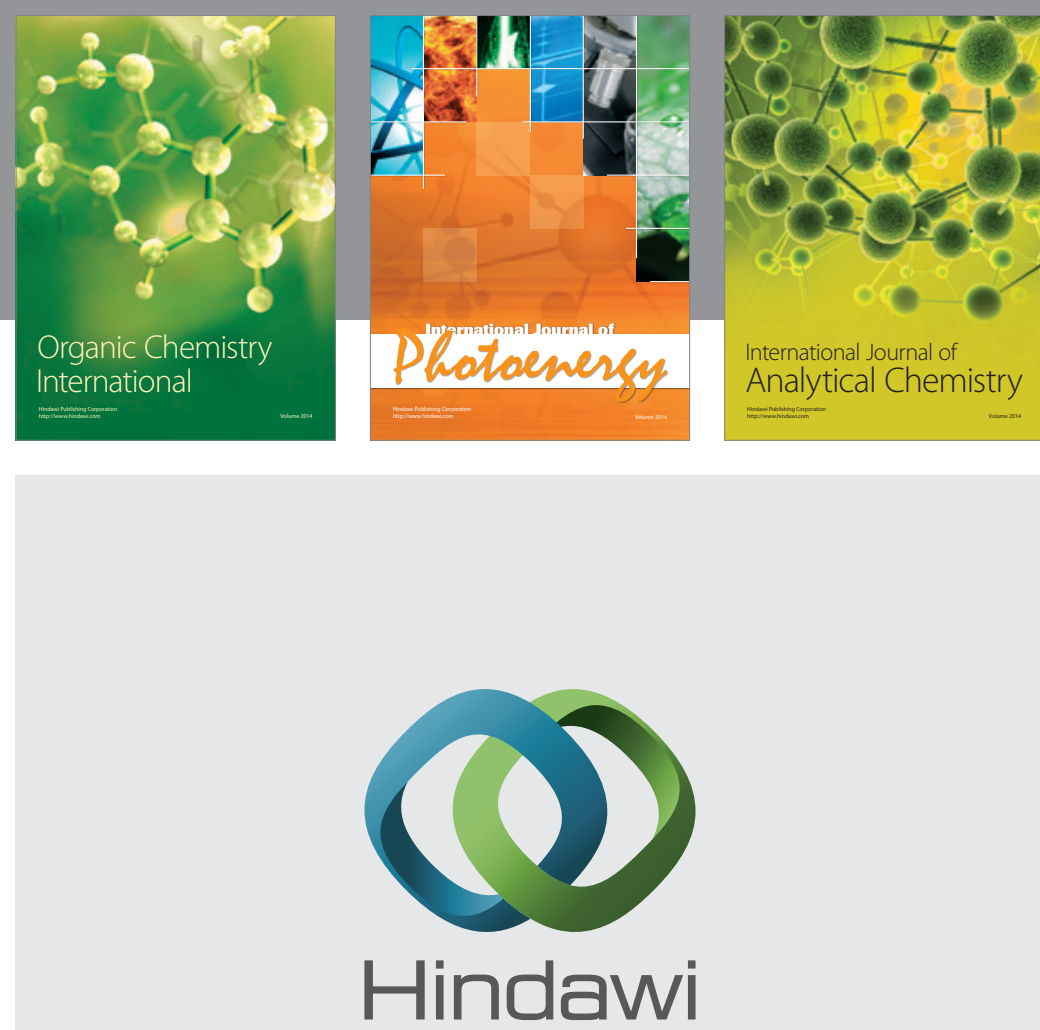

Submit your manuscripts at

http://www.hindawi.com
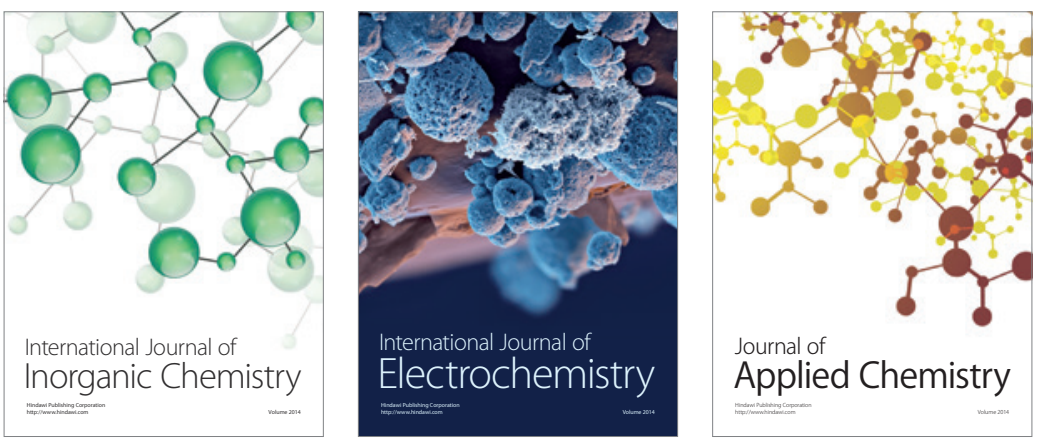

Journal of

Applied Chemistry
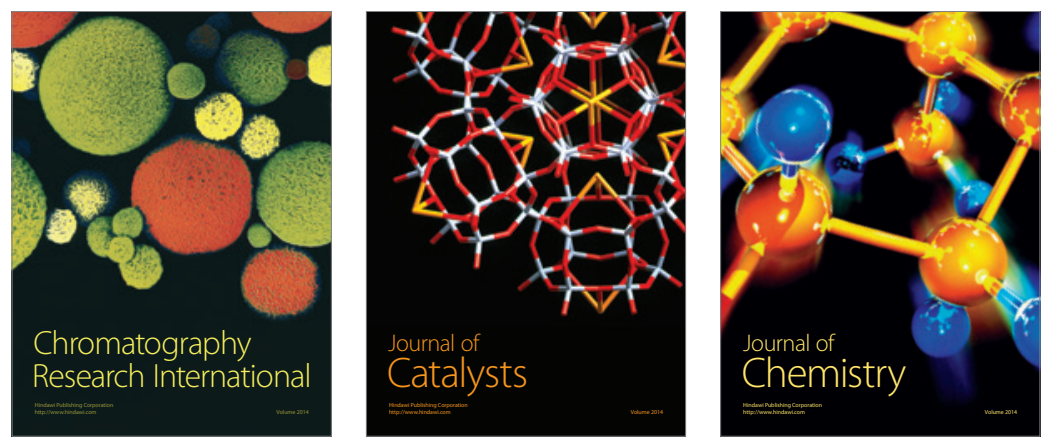
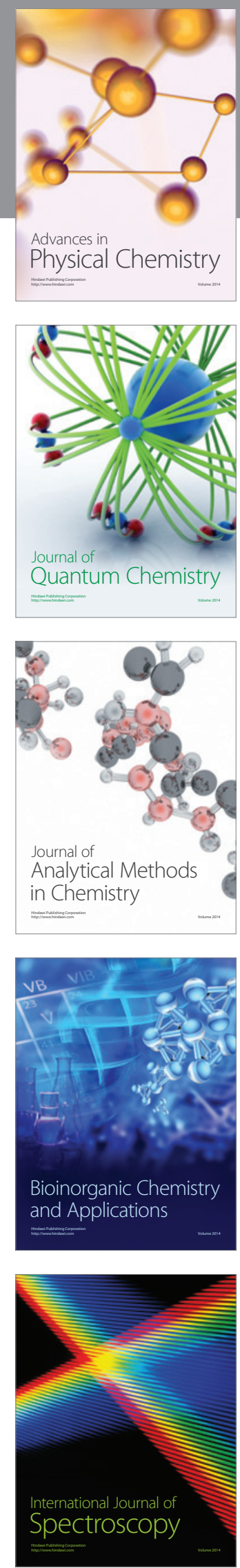